\title{
An American option contract toward supply chain coordination
}

\author{
Masoud Rabbani ${ }^{a^{*}}$, Neda Manavizadeh ${ }^{\mathrm{b}}$, Hamed Vafa Arani ${ }^{\mathrm{a}}$ and Safoura Famil Alamdar
}

${ }^{a}$ School of Industrial Engineering, College of Engineering, University of Tehran, Tehran, Iran ${ }^{b}$ Master of business administrator, Khatam University, Tehran, Iran

\section{H R O N I C L E}

Article history:

Received March 12, 2017

Received in revised format:

October 20, 2017

Accepted December 16, 2017

Available online

December 16, 2017

Keywords:

Supply chain coordination

American option mechanism

Wholesale mechanism

Stochastic programming

Geometric Brownian Motion

\section{Introduction}

Vertically independent decision-making causes a great deal of financial losses for all the members of markets. In essence, vertically independent markets are known as decentralized supply chains (SCs) in which all the members independently transact to satisfy some customers. In other words, SC members act as separated islands and they only take the final decision of the higher layer into account. Moreover, another decentralization origin is derived from personal profit maximization, which results in shrinking the size of the market. In other words, both of these strategies result in local optimum solutions, which make the total profit of the SC lower (Bresnahan \& Reiss, 1985; Lee et al., 1997). Therefore, a mechanism is required to reduce this effect on the performance of the SC. In this regard, coordination mechanisms have been introduced to tackle them. Therefore, in a coordinated SC, the members should not compete as independent entities. In addition, the members of the SC may behave as a part of a unified system (Kanda \& Deshmukh, 2008). Coordination could be achieved with several well-known

\footnotetext{
* Corresponding author

E-mail address: mrabani@ut.ac.ir (M. Rabbani)

(C) 2018 by the authors; licensee Growing Science, Canada.

doi: $10.5267 /$ j.dsl.2017.12.001
}

\begin{abstract}
Coordination improves the profit of all the members in a supply chain. In this paper, a novel coordination mechanism is introduced in a retailer-manufacturer supply chain in which the the market demand throughout a multi-period horizon. The manufacturer follows a make-toorder production policy. This mechanism gives the retailer the right to select the more beneficial mechanism in each period. The retailer as the leader of the chain decides how many options should be purchased at the beginning of the horizon through a mixed-integer mathematical model. This model addresses the uncertainties in the market demand and the
market price, simultaneously. A scenario planning approach is used to treat the random variables within the model. Also, an optimal scenario reduction model is adapted to reduce the computational complexity of the problem. Finally, a numerical experiment is designed to validate the performance of the model. The results demonstrate a remarkable improvement in the profit of both members. Moreover, a number of experiments are performed to show how
the option price, the exercise price and the interest rate affect the performance of the contract.
\end{abstract}

C) 2018 by the authors; licensee Growing Science, Canada. 
mechanisms. We focus on the SC contracts in this study. A supply contract specifies parameters (e.g. quantity, price, quality, and time) for a seller to satisfy the demand of a buyer who aims at increasing the profit of the whole SC (Gan et al., 2005).

In addition, supply contracts could reduce under stock/overstock costs and could share risks among members in addition to increasing total profit of the SC (Tsay, 1999). Thus, structure of the supply contracts is a topic of debate among academicians and practitioners as it could significantly affect the performance of each member. As a result, several types of mechanisms have been addressed in the literature to achieve the prefect SC coordination. Revenue sharing, buyback, and quantity flexibility are the most well-known mechanisms with which perfect coordination could be achieved. Although these contracts could coordinate the supply chain, they may create some consequences influencing the natural function of the market (Chopra \& Meindl, 2007). Therefore, finding a better supply contract has been transformed to a controversial topic among researchers to achieve both the perfect coordination and the least side effects. In this regard, a variety of contracts has been addressed. Sales rebate, delay-in-payments, option contract, etc. are examples of other mechanisms. This study focused on the option contract. An option mechanism has been adopted from financial markets. Because of uncertain nature of financial markets, the most powerful risk-hedging tools have been developed in this area, called derivatives. An option mechanism could mitigate risks associated with market fluctuations and could be used as a coordination mechanism. In essence, an option as a derivative gives the holder of the option the right to buy/sell an asset by a certain date for a certain price (Hull \& Basu, 2016). In other words, the holder of the option buy a right to buy/sell an asset by a maturity date for a fixed exercise price while an initial small premium is required calling an option price. Furthermore, exercise of the option right depends only on the spot price. Although option contract is similar to the original option derivative, there are some major differences among them. In the common option contracts, the buyer buys a right to buy a specified number of real products instead of stock with a maturity date, called selling season instead of maturity date in a fixed exercise price called a wholesale price. Furthermore, exercise condition is existence of the market demand while it is price feasibility in the option mechanism as a derivative (Burnetas \& Ritchken, 2005; Huang, 2009; Gomez Padilla \& Mishina, 2009; Nomikos et al., 2013). To improve the performance of the option contract, other types of the option mechanisms in the financial markets could be helpful. An American option mechanism is adapted to apply in the good markets in this study. It is noteworthy that the basic version of the option mechanism is called a European option. According to an American option mechanism, the holder of the option has the right to exercise the options during the lifetime of the contract while the European one enforces the holder to exercise just at the maturity date. As a result, the American option could improve the European one in several ways. Firstly, the American option makes the contract more flexible compared to the European one. It means that the buyer could experience more degree of freedom to exercise the options during a horizon. Secondly, the American option could be used for products that are more durable. This makes it more applicable since the European can only be used for products with short selling season. Thirdly, price fluctuations could also be considered in an American option while it is ignored in the European one because of the short selling season. It is worth mentioning that there is a significant difference between the American option as a derivative and as a supply contract, which it returns to the exercise rules. In a financial market, the buyer has to exercise all options together while there is not this obligation in the real good market. In a real good market, the buyer can exercise the options in several steps in different periods of the lifetime. Price feasibility is another condition of exercising options in addition to the demand existence. This condition is not addressed by almost all the previous studies. In other words, the previous models enforce the retailer to exercise the options in case of demand existence regardless of the spot market price. As a result, the option might cause additional costs for the retailer instead of risk-hedging. Apart from the American option, a wholesale mechanism is incorporated to the contract to improve the flexibility of the mechanism. The order quantity of the retailer, which is the most critical variable in the option contract, is considered the main decision variable. The sequence of events during the contract is summarized as follows: first, the retailer purchases a number of options with a fixed lifetime, exercise price, and option price. Second, 
the manufacturer produces according to a make-to-order (MTO) strategy. Third, at the beginning of each period, the retailer evaluates two required conditions for option exercising. If the option is feasible, the retailer will exercise the remainder of the options from initial purchased options. It is noteworthy that back order will be occurred if this remainder value is less than the market demand. Otherwise, the retailer will use the wholesale mechanism to provide necessary products. Fourthly, at the end of the contract, the manufacturer could sell the non-exercised options to the market for a salvage value. The current study aims at evaluation of the performance of the proposed contract in comparison with a wholesale contract (as the worst case) and the centralized SC (as the best case). Another approach to choose the feasible mechanism by the retailer is considering contract selection as a decision variable as well as the order quantity of the retailer. In this regard, the optimal solution of the model in a basic and a mechanism selection model will be compared.

The rest of the paper is organized as follows: in section 2, body of the relevant literature is reviewed. In section 3, the basic model and all the extensions to it are presented. Section 4 explains the optimal scenario reduction model as the main part of the solution methodology. Section 5 includes an illustrative numerical experiment to demonstrate applicability of the contract and in the last section, conclusion remarks in addition to some possible extensions to the current study are provided.

\section{Literature review}

Coordination contracts are the most widespread part of the SC coordination literature. Each contract forms a research stream in this area. Although the option contracts have introduced as a coordination mechanism in the last decade, it quickly received much attention of academicians. However, as the focus of the current paper is on the option contracts, the literature is entirely devoted to the option contracts and some compound contracts which involve option mechanism. The previous studies in option contracts usually considered the European option mechanism as the core of their addressed framework in which a two-echelon SC tries to satisfy the final consumer during a single period. Wang \& Liu (2007) introduce a retailer-led option contract in which the retailer aims at coordination of the upstream production quantity. Their results show that the addressed contract improves the profit of both parties of the SC. Gomez Padilla \& Mishina (2009) present an option contract aims at coordination of a two-echelon SC in two cases including a single supplier-single retailer and multiple suppliers-a single retailer. Their results demonstrate improvement in profit of both parties and the whole chain. A cooperative game theory approach is addressed in Zhao et al. (2010) to the option mechanism in which the retailer and the manufacturer make simultaneously the order and production quantity decisions. Aiming at a perfect coordination, they used a wholesale mechanism as a benchmark to select the best parameters of the option contract with a negotiation procedure, which considers the negotiation power and the risk-aversion of the parties. Xia et al. (2011) evaluate the performance of the option contract using a wholesale contract as a benchmark. They show that a supplier with higher disruption risk provides more profit while the option contract could reduce the supplier disruption risks. It can be implied that the SC cannot be perfectly coordinated through the addressed option contract. Zhao et al. (2013) introduce a bidirectional option contract in an MTO production environment with which the retailer can order new product or return unsold products. Chen et al. (2014) presented option contract in which the effects of the risk preferences on the optimal solution of the problem is investigated through considering a risk-neutral supplier and a loss-averse retailer. Hasani et al. (2013) studied the coordination of a supply chain consisting of one vendor and one buyer via the credit option contract. Luoa et al. (2015) studied coordination of a two-stage supply chain consisting of a supplier and a manufacturer with dual procurement sources through real-option contract. In the paper of Cai et al. (2017), an option contract was introduced to coordinate a vendor-managed inventory (VMI) supply chain under yield uncertainty. Chen et al. (2017) studied coordination in a two-echelon supply chain consisting of a supplier and a retailer with bidirectional option contracts and service requirement.

Providing a more flexible contract could be achieved through a combination of several contracts. Some previous studies have addressed compound contracts in which the retailer can simultaneously use all 
of them if some conditions are satisfied. It is worth mentioning that a wholesale mechanism is broadly used to be combined with the option. Cheng et al. (2003) address a mixed wholesale-option contract. In their problem, the buyer purchases a number of products through a wholesale contract in advance and purchases a number of options to exercise at the beginning of the selling season. The authors try to indicate the optimal order quantities for the buyer in both mechanisms and the optimal pricing strategy for the seller. Another hybrid wholesale-option contract is introduced by Burnetas \& Ritchken (2005). In their paper, the retailer can purchase through either an option or a wholesale mechanism. They consider a downward-sloping demand curve. The results demonstrate that a higher uncertainty in demand creates more profit for the retailer. Xu (2010) also proposes a wholesale-option contract considering both the supplier's yield and the market demand as random variables. Buzacott et al. (2011) address a different approach to deal with supply and demand uncertainties in a similar framework to $\mathrm{Xu}$ (2010). Unlike the previous studies, the authors use a mean-variance approach instead of the expected value operator to model the utility of each member. A similar wholesale-option contract is addressed by Chen \& Shen (2012) in which the order quantity of the retailer and the production quantity of the manufacturer have to be decided subject to a minimum service requirement. They show that the retailer's and the supplier's profit are non-increasing and non-decreasing in the service requirement, respectively. Jörnsten et al. (2013) address the wholesale-option mechanism in a random discrete demand environment. They demonstrate that the mixed contract is preferred to a pure option contract when the manufacturer has a bound on how much variance he is willing to accept and is risk-averse. In addition to a wholesale mechanism combined with option contract, other mechanisms have this capability. A vulnerable option contract is introduced by Babich (2006) for the first time in which both parties can defer their decisions. In essence, a vulnerable option is a combination of an option and a postponement mechanism. The author investigates effects of suppliers' default risk and competition on the key decisions of both parties. In addition to the option, forward is another financial derivative, which can be combined with an option contract in SC management. Li et al. (2009) introduce a combination of a forward and an option mechanism to coordinate the SC under demand and price uncertainties and asymmetric information condition. They demonstrate that the forward-option contract dominates other contracts and can eliminate the asymmetric information effects. A combined leasingoption contract is introduced by Liu et al. (2013). In their problem, a carrier and an upstream rental company are considered as parties. The carrier decides quantity of the leased containers, and type and quantity of option contracts to be purchased at the beginning of the contract. Then, the retailer decides quantity of options to be exercised during the selling season. Vafa Arani et al. (2016) introduced a mixed revenue-sharing option contract to coordinate supply chain with a retailer and a manufacturer. To cover drawbacks of the classic contracts, A European call option mechanism and a revenue-sharing mechanism were combined. Yang et al. (2017) studied the call, put and bidirectional option contracts in an agricultural supply chain including one supplier and one retailer where the market demand depends on sales effort.

To the best of our knowledge, previous studies can be criticized in several directions. The current paper tries to cover these drawbacks through its novelties. Firstly, almost all the previous studies used a European option while the American one could improve the European one in several ways. A higher flexibility, a wider application, and considering price fluctuations during the contract are some remarkable advantages of the American mechanism, which is used in this paper for the first time. Secondly, the previous studies used demand existence as only exercise condition for the option mechanism whereas it is not realistic and can cause additional costs for the buyer. In this regard, a price feasibility condition is added to this condition for the first time to improve the performance of the mechanism. Thirdly, as the previous studies addressed a single period selling season, short-term price fluctuations could not be considered in the model although it could have strong effects on the profit of the members. The current study uses a well-known stochastic process namely Geometric Brownian Motion (GBM) to model the short-term fluctuations of the market price. Fourthly, although the basic model accounts the type of contract used by the retailer as an input data, it can be also a decision variable. The model is modified in this way to avoid unreasonable local optimum solutions and to give 
the retailer more authority to choose the more profitable contract. Finally, a comparison between the mixed contract and a wholesale contract, as the nadir solution, as well as a cooperative chain, as the ideal solution, are performed to demonstrate profitability of the contract.

\section{Model description}

Achieving a coordinated SC is one of the most challenging issues among companies. This is because of losses caused by non-coordinated interactions. This paper addresses a new mixed coordination contract using an American option mechanism and a simple wholesale contract to coordinate a retailermanufacturer SC. The market demand and the market price are assumed as independent random variables. The retailer orders a number of products to satisfy the demand throughout the planning horizon. Then, the retailer checks if the American option mechanism is profitable at the beginning of each period. If it is profitable, he will exercise the options up to the initial ordered quantity. Otherwise, the wholesale mechanism will be used. In addition, when all the options are exercised and the wholesale mechanism is not profitable, the retailer prefers not to satisfy the market demand. This causes shortage, which tends to be occurred at the last periods. The manufacturer follows an MTO production policy in which production quantity is equal to the order quantity of the manufacturer. However, the manufacturer is assumed to be able to produce the products in a shorter time to satisfy the orders of the retailer received through the wholesale mechanism at the beginning of each period, called late production. Also, the late production has no limitation. In this case, production costs are higher than the regular production before the selling season. Moreover, the wholesale price of the products is different in these two mechanisms. In the option mechanism, the retailer pays a fee called an option price when he orders products and pays another fee after exercising the options called an exercise price. Nevertheless, in the wholesale mechanism, the retailer pays only a wholesale price, which is assumed a random variable. Since the manufacturer follows an MTO production policy, the only decision variable is the order quantity of the retailer. The described situation in this section is a decentralized $\mathrm{SC}$ because the retailer and the manufacturer act as independent companies. Moreover, they make their decisions without considering the whole chain profit, which is a non-cooperative situation. Consequently, this situation is known as a non-cooperative decentralized SC. In addition, two other situations will be presented and compared. The first one is a centralized chain in which both the retailer and the manufacturer behave as a unified organization. The second one is a cooperative decentralized SC. In this case, although the two parties are independent, they adopt the SC-wide quantity. Nomenclature of the model is presented here.

$T \quad$ Set of planning periods indexed by $t$

$S \quad$ Set of possible scenarios indexed by $S$

$P_{t} \quad$ Unit market price of the product, which follows a GBM with parameters $\left(\mu, \sigma^{2}\right)$

$W_{t} \quad$ Unit wholesale price of the product, which is assumed to be a percent of the market price

$D_{t} \quad$ Market demand of the product, which follows a probability distribution with $F($.$) as$ $\mathrm{CDF}, f($.$) as pdf , F_{c}($.$) as \mathrm{CDF}$ of sums of a number of independent random variables following the same distribution of $f($.

$X_{t} \quad$ The binary variable which shows whether the retailer uses the option mechanism or the wholesale mechanism

c Unit production cost of the product

$b \quad$ Unit shortage cost

$v \quad$ Unit salvage value of the unsold products

$c^{\prime} \quad$ Unit production cost for late production

$r \quad$ Risk-free interest rate

$\operatorname{Pr}_{S} \quad$ Probability of occurrence of scenario $s$

$Q_{t}^{S C} \quad$ Quantity of the centralized SC in period $t$

$Q_{t}^{W R}$ Order quantity of the retailer in a wholesale contract in period $t$

$Q_{t}^{W M}$ Production quantity of the manufacturer in a wholesale contract in period $t$ 
$Q^{D R} \quad$ Order quantity of the retailer in the decentralized SC coordinated with the mixed contract

$Q^{D R S}$ Order quantity of the retailer in the decentralized SC coordinated with the mixed contract in case of mechanism selection

$Q^{D C R}$ Order quantity of the retailer in the cooperative decentralized SC coordinated with the mixed contract

$E C_{t} \quad$ Number of exercised options in period $t$

$E W_{t}$ Number of products provided from the wholesale mechanism in period $t$

$R O_{t} \quad$ Number of non-exercised options in period $t$

To find the optimal solution of the model, a discrete-time mathematical formulation introduces. Although the centralized SC does not exist, it is used as an ideal solution to show the performance of the proposed coordination mechanism. Therefore, firstly, the centralized SC will be formulated and then the non-cooperative decentralized and the cooperative decentralized chain will be considered as more realistic situations. In addition, a simple wholesale contract is presented here as the worst coordination mechanism in which both parties behave completely independently and the interactions are based on the spot prices. The mathematical formulation contains random variables, which should be treated. In other words, the deterministic counterpart of the model should be provided. Sampling, simulation, scenario planning are some methods used for treating randomness of the mathematical models. In this paper, a scenario planning with a scenario reduction method is adopted to provide the deterministic model. Hereafter, index of $s$, which is added to some variables, shows scenario.

\subsection{Centralized supply chain}

When two companies behave as a single identity, a perfect coordination is achieved. There are no losses caused by asymmetric information, double marginalization and shrinking the market in the centralized SC. Therefore, members of the SC should set this situation as a goal to achieve. In the case of the current paper, it is assumed that the centralized organization choose the best order quantity (=production quantity) in order to satisfy the demand of the whole horizon rather than each period. The profit function of the centralized SC is presented in Eq. (1).

$$
\Pi^{S C}=\bar{P} \cdot \min \left\{\sum_{t=1}^{T} D_{t}, Q^{S C}\right\}-c \cdot Q^{S C}-b \cdot\left\{\sum_{t=1}^{T} D_{t}-Q^{S C}\right\}^{+}
$$

Where the first term is the income from selling products to the market. The second one is the production costs and the last term is the shortage costs. As the centralized SC considers the whole horizon, the total demand of the horizon is used in the formulation. In essence, the total demand is a summation of a number of independent random variables. Because it may sell the products to the market in any period of the horizon, an average price is used in Eq. (1). Finally, the closed-form formulation of the optimal order quantity and the expected profit of the chain are presented in Eq. (2) and Eq. (3). Proof of the Eq. (2) is presented in Appendix A.

$$
\begin{aligned}
& \hat{Q}^{S C}=F_{c}^{-1}\left(\frac{\bar{P}+b-c}{\bar{P}+b}\right) \\
& \hat{E}\left[\Pi^{S C}\right]=\sum_{s=1}^{S} P r_{s} \cdot\left[\bar{P} \cdot \min \left\{\sum_{t=1}^{T} D_{t s}, \hat{Q}^{S C}\right\}-c \cdot \hat{Q}^{S C}-b \cdot\left\{\sum_{t=1}^{T} D_{t s}-\hat{Q}^{S C}\right\}^{+}\right]
\end{aligned}
$$

\subsection{Wholesale contract}

In the wholesale mechanism, each company either produces or purchases products according to the private information. In this situation, the retailer orders according to the realized market demand and purchases the products in a wholesale price. The manufacturer produces according to the market demand. Because the manufacturer produces in a shorter period at the beginning of each period, the production costs are higher than the regular production before the beginning of the horizon. Eq. (4) and Eq. (5) show the profit functions of the retailer and the manufacturer in the wholesale mechanism. 


$$
\begin{aligned}
& \Pi_{t}^{R W}=P_{t} \cdot \min \left\{D_{t}, Q_{t}^{W R}\right\}-W_{t} \cdot Q_{t}^{W R}-b \cdot\left\{D_{t}-Q_{t}^{W R}\right\}^{+}+v \cdot\left\{Q_{t}^{W R}-D_{t}\right\}^{+} \\
& \Pi_{t}^{M W}=W_{t} \cdot \min \left\{D_{t}, Q_{t}^{W M}\right\}-c^{\prime} \cdot Q_{t}^{W M}-b \cdot\left\{D_{t}-Q_{t}^{W M}\right\}^{+}+v \cdot\left\{Q_{t}^{W M}-D_{t}\right\}^{+}
\end{aligned}
$$

where, in both equations, the first term is the income, the second term is cost of products, the third term is shortage cost and the last term is the income from selling extra products. Finally, closed-form solutions of the strategies of the parties in this mechanism are expressed in Eqs. (6) and (7). See appendix A for proof. Also, after substitution of them to the profit functions, the optimal expected profit of the retailer and the manufacturer are presented in Eq. (8) and Eq. (9).

$$
\begin{aligned}
& \hat{Q}_{t s}^{W R}=F^{-1}\left(\frac{P_{t s}+b-W_{t s}}{P_{t s}+b-v}\right) \\
& \hat{Q}_{t s}^{W M}=F^{-1}\left(\frac{W_{t s}+b-c}{P_{t s}+b-v}\right) \\
& \hat{E}\left[\Pi^{W R}\right]=\sum_{t=1}^{T}\left(\sum_{s=1}^{S} P r_{s} \cdot\left[P_{t s} \cdot \min \left\{D_{t s}, \hat{Q}_{t s}^{W R}\right\}-W_{t s} \cdot \hat{Q}_{t s}^{W R}-b \cdot\left\{D_{t s}-\hat{Q}_{t s}^{W R}\right\}^{+}+v \cdot\left\{\hat{Q}_{t s}^{W R}-D_{t s}\right\}^{+}\right] \cdot e^{-r \cdot t}\right) \\
& \hat{E}\left[\Pi^{W M}\right]=\sum_{t=1}^{T}\left(\sum_{s=1}^{S} \operatorname{Pr}_{s} \cdot\left[W_{t s} \cdot \min \left\{D_{t s}, \hat{Q}_{t s}^{W M}\right\}-c^{\prime} \cdot \hat{Q}_{t s}^{W R}-b \cdot\left\{D_{t s}-\hat{Q}_{t s}^{W M}\right\}^{+}+v \cdot\left\{\hat{Q}_{t s}^{W M}-D_{t s}\right\}^{+}\right] \cdot e^{-r \cdot t}\right)
\end{aligned}
$$

The wholesale contract is used as a nadir solution to the problem, which can show the performance of the proposed mechanism in terms of raising the profit of parties and that of the whole chain.

\subsection{Non-cooperative decentralized supply chain}

In most of real situations, companies act as independent identities. Also, they usually do not have a chain-wide insight in their decisions. This situation is a non-cooperative decentralized SC in which companies need a coordination mechanism to avoid losses associated with decentralization. The basic model is presented here.

\subsubsection{The basic model}

As mentioned before, the manufacturer follows an MTO production policy in which the products are produced only after receiving an order from the retailer. Although the manufacturer can produce quickly at the beginning of each period, it also depends on the order quantity of the retailer through the wholesale mechanism. Therefore, the most critical decision is the order quantity of the retailer. The retailer will use the option mechanism if the wholesale price exceeds the discounted total value of the option price and the exercise price of the options. Otherwise, the retailer will provide the products through the wholesale mechanism. Eq. (10) shows the logical condition for mechanism selection.

$$
X_{t}=\left\{\begin{array}{l}
1, W_{t}>e p+o \cdot e^{r t} \\
0, W_{t} \leq e p+o \cdot e^{r t}
\end{array}\right.
$$

The retailer aims at maximization of his profit. Therefore, the order quantity will be set on an amount to satisfy optimally conditions. Eq. (11) and Eq. (12) show the initial profit and the periodic profit of the retailer, respectively.

$$
\begin{aligned}
& \Pi_{0}^{R}=-o \cdot Q \\
& \Pi_{t}^{R}=P_{t} \cdot \min \left\{D_{t}, E C_{t}+E W_{t}\right\}-e p \cdot E C_{t}-W_{t} \cdot E W_{t}-b .\left\{D_{t}-E C_{t}-E W_{t}\right\}^{+}
\end{aligned}
$$

where Eq. (11) is the option price paid for purchased options. In the Eq. (12), the first term is the income from selling products to the final market. The second one is exercise price paid for exercised options. $E C_{t}$ represents the amount of options which the retailer exercises in each period. It is defined in Eq. (13).

$$
E C_{t}=X_{t} \cdot \min \left\{D_{t}, R O_{t}\right\}^{+}
$$

where $R O_{t}$ represents the remaining amount of options, which is shown in Eqs. (14) and (15). 


$$
\begin{aligned}
& R O_{0}=Q \\
& R O_{t}=R O_{t-1}-E C_{t}
\end{aligned}
$$

The third term of Eq. (12) is the wholesale price of the products purchased through the wholesale mechanism, which is defined in Eq. (16).

$$
E W_{t}=\left(1-X_{t}\right) \cdot D_{t}
$$

The last term of the Eq. (12) is the costs of shortage. Finally, the total profit of the retailer is calculated with adding the initial profit and the net present value of the periodic profits as shown in Eq. (17).

$$
\Pi^{R}=\sum_{t=1}^{T}\left(\Pi_{t}^{R} \cdot e^{-r t}\right)-o \cdot Q
$$

According to the equations, the model contains not only random variables, but also nonlinear terms. To achieve an optimal solution, firstly, a scenario planning approach is used to treat the random variables. Secondly, the model is linearized with defining some auxiliary variables and adding some extra constraints. $\alpha_{t s}$ is defined as $\min \left\{D_{t}, E C_{t}+E W_{t}\right\}$. In essence, $\alpha_{t s}$ is the sale amount of the retailer. Also, $\beta_{t s}$ is defined as $\min \left\{D_{t}, R O_{t}\right\}^{+}$, that shows the exercisable amount of options in each period. Finally, $\delta_{t s}$ is defined as $\left\{D_{t}-E C_{t}-E W_{t}\right\}^{+}$, which shows amount of shortage in each period. The resulted linearized mathematical formulation is presented in Eqs. (18)-(26).

P1: $\max \quad E\left[\Pi^{R}\right]=\sum_{t=1}^{T}\left(\sum_{s=1}^{S} P r_{s}\left[P_{t s} \cdot \alpha_{t s}-e p \cdot X_{t s} \cdot \beta_{t s}-W_{t s} \cdot\left(1-X_{t s}\right) \cdot D_{t s}-\right.\right.$

b. $\left.\left.\delta_{t s}\right] \cdot e^{-r t}\right)-o \cdot Q^{D R}$

subject to:

$$
\begin{array}{lc}
\alpha_{t s} \leq D_{t s} & \forall t, s \\
\alpha_{t s} \leq X_{t s} \cdot \beta_{t s}+\left(1-X_{t s}\right) \cdot D_{t s} & \forall t, s \\
\beta_{t s} \leq D_{t s} & \forall t, s \\
\beta_{t s} \leq R O_{t s} & \forall t, s \\
R O_{t s}=R O_{(t-1) s}-X_{t s} \cdot \beta_{t s} & \forall t, s \\
R O_{0 s}=Q^{D R} & \forall t, s \\
\delta_{t s} \geq D_{t s}-X_{t s} \cdot \beta_{t s}-\left(1-X_{t s}\right) \cdot D_{t s} & \forall t, s \\
\alpha_{t s}, \beta_{t s}, \delta_{t s}, Q^{D R} \geq 0 & \forall t, s
\end{array}
$$

Eq. (19) and Eq. (20) guarantee exact definition of $\alpha_{t s}$. Eq. (21) and Eq. (22), similarly, guarantee the definition of $\beta_{t s}$. Eq. (23) defines the remaining amount of options, namely $R O_{t s}$. Eq. (24) shows that the initial amount of the options is equal to the order quantity of the retailer. Eq. (25) guarantees the exact definition of $\delta_{t s}$ and Eq. (26) is non-negativity constraint.

Although the manufacturer does not make any decision in the model, his expected profit is important to show the performance of the proposed mechanism. Eq. (27) shows the expected profit of the manufacturer.

$$
\begin{aligned}
& E\left[\Pi^{s}\right]=\sum_{t=1}^{T} P r_{s}\left[\sum_{s=1}^{S} e p \cdot X_{t s} \cdot \widehat{\beta_{t s}}+\left(W_{t s}-c^{\prime}\right) \cdot\left(1-X_{t s}\right) \cdot D_{t s}\right] \cdot e^{-r t}+o \cdot \widehat{Q}^{D R}-c \cdot \widehat{Q}^{D R}+ \\
& v \cdot \widehat{R O}_{T} \cdot e^{-r T}
\end{aligned}
$$

where the first term is the exercise costs of the options paid by the retailer. The second term is profit of the manufacturer in the wholesale mechanism. The third one introduces the option price of the options. The fourth term is production costs and the last one is salvage value of the remaining options at the end of the horizon. It is worth mentioning that the total profit of the chain is summation of $E\left[\Pi^{R}\right]$ and $E\left[\Pi^{s}\right]$. 


\subsubsection{The mechanism selection model}

The basic model uses the logical condition presented in Eq. (10) to adopt the better mechanism. However, it might be better to let the model choose the better mechanism. In this regard, $X_{t}$ is replaced by a binary variable $Y_{t s}$. In other words, the modified model suggests the companies to use either the American option mechanism or the wholesale mechanism. The modified model could be reformulated as follows:

$$
\begin{array}{lc}
\text { P2: } \max E\left[\Pi^{R}\right]=\sum_{t=1}^{T}\left(\operatorname { P r } _ { s } \left[\sum_{s=1}^{S} P_{t s} \cdot \alpha_{t s}-e p \cdot Y_{t s} \cdot \beta_{t s}-W_{t s} \cdot\left(1-Y_{t s}\right) \cdot D_{t s}-\right.\right. & \\
\left.\left.b . \delta_{t s}\right] \cdot e^{-r t}\right)-o \cdot Q & \forall t, s \\
\text { subject to } & \forall t, s \\
\alpha_{t s} \leq D_{t s} & \forall t, s \\
\alpha_{t s} \leq Y_{t s} \cdot \beta_{t s}+\left(1-Y_{t s}\right) \cdot D_{t s} & \forall t, s \\
\beta_{t s} \geq D_{t s} & \forall t, s \\
\beta_{t s} \geq R O_{t s} & \forall t, s \\
R O_{t s}=R O_{(t-1) s}-E C_{t s} & \forall t, s \\
R O_{0}=Q^{D R S} & \forall t, s \\
\delta_{t s} \geq D_{t s}-Y_{t s} \beta_{t s}-\left(1-Y_{t s}\right) \cdot D_{t s} & \\
\alpha_{t s} \beta_{t s}, \delta_{t s}, Q \geq 0 & \\
Y_{t s} \in\{0,1\} . &
\end{array}
$$

Despite the previous linearization in the basic model, the modified model is nonlinear. $Y_{t s} \cdot \beta_{t s}$ is a multiplication of two variables which make the model a nonlinear one. In this regard, the model is linearized by replacing $Y_{t s} . \beta_{t s}$ by $Z_{t s} \geq 0$ and adding the following constraints.

$$
\begin{array}{lr}
Z_{t s} \leq M \cdot Y_{t s}, & \forall t, s \\
Z_{t s} \leq M \cdot \beta_{t s}, & \forall t, s \\
Z_{t s} \leq \beta_{t s}-M \cdot\left(1-Y_{t s}\right) . & \forall t, s
\end{array}
$$

\subsection{Decentralized cooperative supply chain}

In many cases, it is impossible for members of a SC to act as a unified organization. Therefore, they choose other actions to achieve a coordinated chain. Using the optimal quantity of the centralized SC is a strategy to achieve a better coordination situation. This is shown in Eq. (40). The optimal order quantity of the centralized SC should be substituted in Eqs. (18) and (27) to calculate the profit of both parties in this situation.

$$
Q^{D C R}=\hat{Q}^{S C} \text {. }
$$

\subsection{Price modeling (GBM)}

As discussed before, the market price of products is a random variable. Since the proposed mechanism is designed in a multiple period environment, a stochastic process is adopted to model the random price during the horizon. One of the most well-known stochastic processes in price modeling is Geometric Brownian Motion (GBM). Although the GBM is known as a continuous-time stochastic process, a discrete-time version is used in more realistic situations. According to a GBM, change in price is calculated as Eq. (41).

$$
d P=\mu \cdot P(0) \cdot d t+\sigma \cdot P(0) \cdot d z
$$


where $P(0)$ is the initial spot price of the product, $\mu$ and $\sigma$ represent the mean value and volatility of the market price. Also $d z=\varepsilon \cdot \sqrt{d t}$, so:

$$
\frac{d P}{P(0)}=\mu \cdot d t+\sigma \cdot \varepsilon \cdot \sqrt{d t},
$$

where $\varepsilon$ is a standard normal random number. The left hand side of Eq. (42) is the change in price in a short period, and the right hand side includes $\mu . d t$ as expected value of this return and the stochastic component of the return. Therefore, change in price follows a normal distribution with mean of $\mu$. $d t$ and standard deviation of $\sigma^{2} . d t$. Let $P(t)$ be the market price in period $t$, it can be calculated with the following equation:

$$
P(t)=P(0)(1+\mu \cdot d t+\sigma \cdot \varepsilon \cdot \sqrt{d t}) .
$$

Finally, the discrete-time version of the price is calculated in Eq. (44), which is used in the current model.

$$
P_{t}=P_{0}(1+\mu \cdot \Delta t+\sigma \cdot \varepsilon \cdot \sqrt{\Delta t}) . \quad \forall t
$$

\section{Scenario reduction}

There are several parameters in a real problem that contains a level of uncertainty. These problems are called stochastic programming models. Uncertainty is modeled through either continuous probability distributions or discrete ones. The current problem faces uncertainty in the market demand, which follows a normal distribution, and uncertainty in the market price, which follows a GBM. Therefore, a computational approach is required to treat such complicated random variables. The size of the approximated model is strongly related to the number of scenarios generated for approximation (Dupačová et al., 2003). Scenario reduction is an approach that facilitates solving the problem through reducing the number of scenarios. There are several previous studies which present different methods and algorithms to reduce the number of generated scenarios (Dupačová et al., 2003; Niknam et al., 2012; Wang, 2010; Watkins et al., 2000; Zhang \& Wang, 2009; Heitsch \& Römisch, 2003; Gröwe Kuska, 2003). In essence, a scenario reduction is a selection problem. In other words, a number of scenarios will be selected and others will be removed if some criteria are satisfied. Two main groups of these criteria are matching statistical moments and distances (Li \& Floudas, 2014). An optimal scenario reduction method has recently introduced by Li \& Floudas (2014). This method is adapted in the current study to reduce the number of generated scenarios. This method applies several criteria to quantify not only the difference in the distribution of the parameter input, but also the difference in the output of the model. They used four criteria in the objective function of the mathematical model to achieve the best set of scenarios. The first one is the Kantorovich distance between the probability distribution. The second one is the difference between the expected objective values. The third one is the difference between the best objective values, and the last one is the difference between the worst objective values. The objective function of the whole scenario reduction model is presented in Eq. (45).

$$
\min \sum_{s=1}^{S} \operatorname{Pr}_{s}^{\text {orig }} d_{s}+Z_{\max }^{\text {err }}+Z_{\min }^{\text {err }}+Z_{\text {exp }}^{\text {err }}
$$

where $d_{s}$ is the Kantorovich distance and its definition is expressed in Eq. (46). Also, other components of the objective function are defined in Eqs. (47)-(49).

$$
\begin{aligned}
& d_{s}=\min _{s \prime} \sum_{t=1}^{T}\left|D_{t s}-D_{t s^{\prime}}\right|+\sum_{t=1}^{T}\left|P_{t s}-P_{t s^{\prime}}\right|+\left|Z_{s}^{*}-Z_{s^{\prime}}^{*}\right|, \\
& Z_{\text {exp }}^{\text {err }}=\left|Z_{\text {exp }}^{\text {New }}-Z_{\text {exp }}^{\text {Orig }}\right|, \\
& Z_{\text {max }}^{\text {err }}=Z_{\text {max }}^{\text {Orig }}-Z_{\text {max }}^{\text {New }}, \\
& Z_{\text {min }}^{\text {err }}=Z_{\text {min }}^{\text {Orig }}-Z_{\text {min }}^{\text {New }},
\end{aligned}
$$


where $S^{\prime}$ is also indexed of scenarios. $Z_{\text {exp }}^{\text {New }}$ and $Z_{\text {exp }}^{\text {Orig }}$ are expected objective value among the original set of scenarios and the selected set of scenarios, respectively. Similarly, $Z_{\text {max }}^{\text {Orig }}$ and $Z_{\text {max }}^{\text {New }}$ are the maximum objective value among the original set of scenarios and the selected set of scenarios, respectively. Also, $Z_{\mathrm{min}}^{\mathrm{Orig}}$ and $Z_{\mathrm{min}}^{\mathrm{New}}$ are the minimum objective value among the original set of scenarios and the selected set of scenarios, respectively. The whole model, which is formulated as a mixed-integer programming model, is presented in Appendix B.

\section{Numerical experiments}

A numerical experiment can validate the performance of the model. Moreover, several analyses based on the numerical experiment provide some insight for the decision makers. Therefore, a numerical experiment is presented in this section in which data are randomly generated. Although the data are not realistic, all the main assumptions and initial conditions are met. The market demand, the market price, the wholesale price, the option price, and cost coefficients are required as inputs of the system. In this experiment, the market demand is assumed to follow a normal distribution with mean of 50 and standard deviation of 10 . The market price follows a GBM with drift parameter of 0.75 and volatility of 0.5 with initial price of 5 . The wholesale price is assumed $80 \%$ of the market price. The option price and the exercise price of options are assumed 0.6 and 4.5 , respectively. The unit production cost is 3 in regular time. However, the unit production cost at the beginning of each period, namely late production, is 4 . The salvage value of the extra products at the end of the horizon is 2 and, finally, the annual interest rate is 0.3 . Also, the planning horizon last 10 periods.

\subsection{Scenario reduction}

There are two main random variables in the optimization model. The first one is the market demand following a normal distribution with parameters 50 and 10. The second one is the market price which follows a GBM with parameters 0.75 and 0.5 and initial price of 5 . In order to make the best equal discrete distribution, a large number of scenarios are generated with Monte Carlo method. Although a large number of scenarios create a better approximation, it can make the problem computationally hard to solve. Therefore, the described optimal scenario reduction model is utilized to choose some scenarios that have the same statistical characteristics of the main set of scenarios. In this regard, firstly, 750 scenarios are generated. Then, the number of scenarios reduces to 50 scenarios with the scenario reduction method. Table 1 shows the descriptive statistics of the original and the new set of scenarios. Maximum value, minimum value, expected value, standard deviation, skewness and kurtosis of each case are presented in this table. According to the table, despite removing $93.3 \%$ of the original scenarios, the main statistics remain close to the original set. Therefore, the new set of scenarios will be used as input arguments of the model.

Table 1

Descriptive statistics of the original and the new set of scenarios

\begin{tabular}{cccccccc}
\hline Parameter & size & max & Min & Average & Standard deviation & Skewness & Kurtosis \\
\hline The market & 750 & 92.18 & 16.48 & 49.88 & 9.918729 & -0.0058 & -0.07064 \\
demand & 50 & 76.55 & 19.61 & 49.71 & 9.44923 & -0.08016 & 0.070577 \\
\hline $\begin{array}{c}\text { The market } \\
\text { price }\end{array}$ & 750 & 19.12 & 0.33 & 8.87 & 2.451302 & -0.13007 & 0.109139 \\
\hline
\end{tabular}

\subsection{Results}

After selecting the best set of scenarios among the original set of scenarios and providing corresponding probability of each new scenarios by means of the optimal scenario reduction model, the main outputs of the model are presented in this section. As the planning horizon starts, the retailer also starts to evaluate the market conditions if it is possible to exercise options. In essence, the remaining quantity of the options is the state variable of the problem. Fig. 1 shows the remaining quantity of the options during the planning horizon in one of the 50 possible scenarios. Also, the exercised quantity of the 
options is contained in this figure. The figure validates the performance of the model. In periods 2 and 6 , the retailer finds the option mechanism less profitable than the wholesale mechanism. Consequently, the exercised quantity is equal to zero and the remaining quantity curve is horizontal during these periods. At the end of the horizon, the discrepancy between the remaining and the exercised quantity of options is the extra quantity of products, which the manufacturer sells them in the salvage value. As mentioned in section 2, the SC could be formed in three situations. These situations include the centralized SC as the perfect coordination mechanism, the non-cooperative decentralized and the cooperative decentralized SC. In addition, the wholesale contract is addressed as the worst solution to the problem. In this section, a comparison between the proposed mixed coordination mechanism and other possible situations is performed. The optimal order quantity of the retailer, corresponding profit of each party, and the total profit of the SC are provided in Table 2 to show the differences.

Following points are results of the comparison performed in Table 2.

- The proposed mixed coordination mechanism remarkably improves the profit of both the retailer and the manufacturer compared to the wholesale mechanism. Therefore, the new mixed contract can be used by practitioners to provide more profit for all the parties and the whole SC,

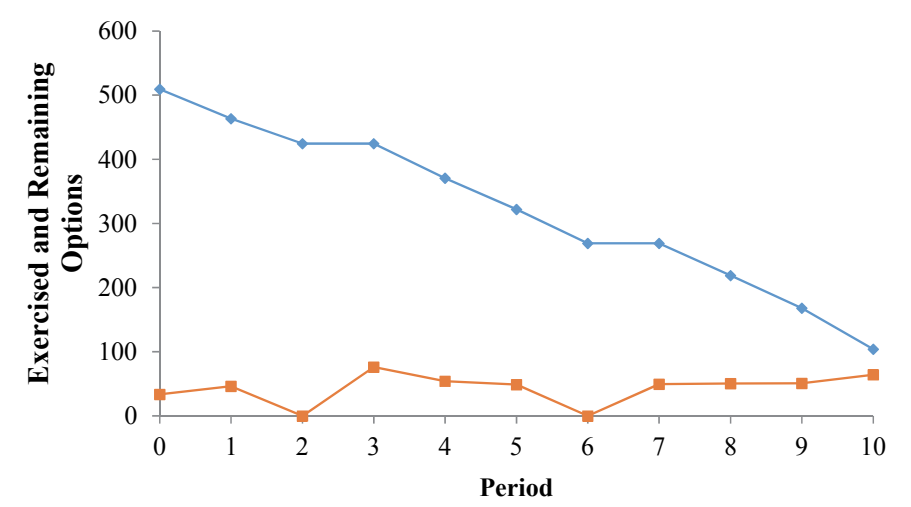

Fig. 1. The exercised and the remaining quantity of options during the horizon

Table 2

A comparison between different situations of the SC

\begin{tabular}{lcccc}
\hline & Wholesale & $\begin{array}{c}\text { Centralized } \\
\text { (Cooperative) }\end{array}$ & $\begin{array}{c}\text { Non-cooperative } \\
\text { Decentralized }\end{array}$ & $\begin{array}{c}\text { Cooperative } \\
\text { Decentralized }\end{array}$ \\
\hline Order Quantity & - & 582 & 509 & 582 \\
Retailer's Profit & 429.637 & - & 1664.63 & 1618.176 \\
Manufacturer's Profit & 736.511 & - & 1285.748 & 1459.192 \\
Total Profit & 1166.148 & 4284.688 & 2950.378 & 3077.368 \\
\hline
\end{tabular}

- The centralized company orders (or produces) more products than the independent retailer in the non-cooperative decentralized situation. Therefore, the centralized company can satisfy a greater portion of the market demand. The more the SC coordinated, the more demand is satisfied and the more profit gained,

- Choosing the centralized strategy in the decentralized SC can slightly improve the profit of the whole SC. While the retailer's profit is decreased, the manufacturer's profit is increased. Therefore, the cooperative decentralized situation is more profitable for the manufacturer because this strategy reduces the power of the retailer as the leader.

- The proposed mixed mechanism cannot achieve a perfect coordination. This is a result of comparison between profit of the cooperative centralized SC and that of the decentralized noncooperative $\mathrm{SC}$,

- Finally, the companies should try to achieve a centralized SC. If it is not possible, they can use the proposed coordination mechanism in this paper to reduce losses of decentralization. 


\subsection{Sensitivity analysis}

In addition to the results of the previous section, which show the remarkable profit improvement created by the proposed coordination mechanism, some analyses on the critical parameters of the model are beneficial to show the reasonable performance of the model. The option and the exercise price are the most important parameters of the model, which have remarkable effects on mechanism selection in the proposed mixed contract. In financial markets, there are standard couples of the option and the exercised price that the companies offer to buyers. This causes considering them as parameters rather than decision variables, but sensitivity analysis helps the decision makers to choose the best couple of the option and the exercise price.

\subsubsection{Option price}

An option price is a fee that the retailer pays to the manufacturer at the beginning of the contract. Since the retailer exercise options according to the market demand and the market price, it is possible that a great deal of options do not be exercised. Therefore, the retailer should minimize the number of options. On the other hand, the option mechanism offers a predetermined constant price, which mitigates the risk of increased market price and motivate the retailer to buy more options. Thus, the option and the exercise price are the most important parameters of the proposed mixed mechanism. In this section, effects of the option price on the outputs of the model are investigated.

Table 3

Effects of the option price on output of the model

\begin{tabular}{ccccc}
\hline Option Price & Order Quantity & Retailer's Profit & Manufacturer's Profit & Total Profit \\
\hline 0 & 602 & 2001.388 & 1087.732 & 3089.12 \\
0.2 & 560 & 1883.087 & 1155.371 & 3038.458 \\
0.4 & 526 & 1771.524 & 1213.033 & 2984.557 \\
0.6 & 509 & 1664.63 & 1285.748 & 2950.378 \\
0.8 & 469 & 1560.304 & 1313.637 & 2873.941 \\
1 & 441 & 1467.424 & 1352.39 & 2819.814 \\
\hline
\end{tabular}

Table 3 shows changes in the retailer's profit, the manufacturer's profit and the order quantity of the retailer in different values of the option price between 0 and 1 . The interval is selected according to $o+v<c$, which avoids arbitrage with the option by the manufacturer. The following remarks are provided according to the Table 3 . If the exercise price and other parameters are kept constant,

- The profit of the manufacturer will be increased. This is obvious because the manufacturer gain a higher income from the selling options,

- In addition, the retailer's profit will be decreased. This is, similarly, logical because the retailer pays more money for each option,

- The order quantity of the retailer will be decreased. This is because of the fact that the option mechanism becomes less profitable for the retailer. Therefore, the number of purchased options will be decreased and the retailer prefers to use the wholesale mechanism, and

- The profit of the whole SC will also be decreased because the retailer prefers the wholesale mechanism and this will decrease the profit of the whole SC as shown in Table 2.

\subsubsection{Exercise price}

An exercise price is the main part of the cost of the product that the retailer will pay if he exercises an option. Therefore, it plays an effective role in the option mechanism. In this section, the exercise price changes between 3 and 6.5. The lower bound is set because of the logical constraint $o+v<e p$, which avoids an unreasonable situation in which the manufacturer prefers to sell the products in salvage value rather than selling to the retailer. The upper bound is selected so that avoids a situation in which the retailer always uses one of the mechanisms in the mixed contract. Table 4 shows changes in the profit of members, profit of the whole SC, and the optimal order quantity of the retailer. 
It is obvious that the trends of the outputs are similar to those caused by the change in the option price. Also, similar logical reasons could be provided for these trends. However, there are some differences between them. Following results are provided to shed a light on these differences.

- The exercise price plays a more effective role in the optimal profits. This is because the exercise price is usually bigger than the option price.

- The exercise price has a less effective role on the order quantity of the retailer. A comparison between Table 3 and Table 4 shows that an increase of one unit in the option price causes a 160unit decrease in the order quantity, while an increase of one unit in the exercise price causes a 64-unit decrease in the order quantity. This is because of the direct effect of the option price on the profit of the retailer. In other words, the manufacturer could tolerate an increase in the exercise price with not exercising the purchased options, but it is inevitable in case of an increase in the option price.

- There are couples of the option and the exercise price that create the same profit. Some couples of $(o, e)$ are presented in Table 5. If the decision makers consider a particular level of the profit of the chain, several couples of $(o, e)$ could be suitable. In other words, there are some indifference curves on each of which several couples of $(o, e)$ provide an equal level of profit. Shaded numbers in the table show three couples of $(o, e)$ which provide approximately an equal profit.

It is worth mentioning that the indifference curves are decreasing. It means that on a particular curve, an increase in the exercise price caused a decrease in the option price.

\section{Table 4}

Effects of the exercise price on output of the model

\begin{tabular}{ccccc}
\hline Exercise Price & Order Quantity & Retailer's Profit & Manufacturer's Profit & Total Profit \\
\hline 3 & 555 & 2303.397 & 756.49 & 3059.887 \\
3.25 & 550 & 2190.551 & 853.702 & 3044.253 \\
3.5 & 550 & 2076.999 & 958.748 & 3035.747 \\
3.75 & 537 & 1969.591 & 1036.172 & 3005.763 \\
4 & 527 & 1870.122 & 1122.185 & 2992.307 \\
4.25 & 511 & 1765.487 & 1195.249 & 2960.736 \\
4.5 & 509 & 1664.63 & 1285.748 & 2950.378 \\
4.75 & 482 & 1568.11 & 1333.945 & 2902.055 \\
5 & 458 & 1472.553 & 1368.016 & 2840.569 \\
5.25 & 454 & 1384.777 & 1440.989 & 2825.766 \\
5.5 & 434 & 1304.662 & 1489.028 & 2793.69 \\
5.75 & 406 & 1226.543 & 1518.914 & 2745.457 \\
6 & 390 & 1157.498 & 1557.196 & 2714.694 \\
6.25 & 368 & 1083.941 & 1577.521 & 2661.462 \\
6.5 & 330 & 1025.924 & 1565.176 & 2591.1 \\
\hline
\end{tabular}

Table 5

Profit of the whole SC in different values of option and exercise price

\begin{tabular}{cccccc}
\hline & \multicolumn{5}{c}{ Exercise price } \\
\cline { 2 - 6 } Option Price & 3 & 4 & 5 & 6 & 7 \\
\hline 0 & 3195.98 & 3153.876 & $\mathbf{3 0 7 0 . 2 6 6}$ & 3001.064 & 2837.107 \\
0.25 & 3106.514 & $\mathbf{3 0 7 1 . 4 9 7}$ & 2967.962 & 2828.039 & 2687.854 \\
0.5 & $\mathbf{3 0 6 6 . 1 8 5}$ & 3006.829 & 2896.98 & 2757.432 & 2529.521 \\
0.75 & 3050.331 & 2967.49 & 2823.662 & 2657.434 & 2446.205 \\
1 & 2997.14 & 2919.306 & 2748.942 & 2527.707 & 2408.575 \\
\hline
\end{tabular}

\subsubsection{Interest Rate}

Unlike the previous studies, this study uses a multiple period framework to the coordination problem. Therefore, the time value of money becomes a significant factor in the profit functions. Moreover, different countries have different interest rates. Thus, different interest rates should be considered to 
make the best decisions for different countries. Table 6 shows the profit of the members, profit of the whole SC and the order quantity in different values of interest rate. According to the table 6 , following remarks can be provided

- The interest has a reverse effect on all the outputs of the model. The decrease in profits is an obvious result of decreased present value of the profit caused by higher level of the interest rate.

- The logical condition of mechanism selection, presented in Eq. (10), depends on the interest rate. Although the short planning horizon hides the effects of the interest rate to some extent, the order quantity decreases slightly in higher values of interest rate. Therefore, higher values of interest rate encourage the retailer to use the wholesale mechanism. This decreases the profit of the members and that of the whole SC (see Table 2).

Table 6

Effects of the interest rate on outputs of the model

\begin{tabular}{ccccc}
\hline Annual Interest Rate & Order Quantity & Retailer's Profit & Manufacturer's Profit & Total Profit \\
\hline 0 & 523 & 1987.505 & 1683.599 & 3671.104 \\
0.05 & 518 & 1929.219 & 1603.388 & 3532.607 \\
0.1 & 511 & 1871.775 & 1521.875 & 3393.65 \\
0.15 & 511 & 1817.477 & 1460.294 & 3277.771 \\
0.2 & 511 & 1766.256 & 1401.296 & 3167.552 \\
0.25 & 509 & 1714.5 & 1341.126 & 3055.626 \\
0.3 & 509 & 1664.63 & 1285.748 & 2950.378 \\
0.35 & 509 & 1616.907 & 1231.966 & 2848.873 \\
0.4 & 496 & 1569.987 & 1161.663 & 2731.65 \\
\hline
\end{tabular}

\subsubsection{Mechanism selection}

In the basic model, the mechanism is selected by a logical condition, while the mechanism could be adopted by the mathematical model as a decision variable. Existence of shortage may disturb the function of the logical condition of the basic model. A comparison between results of these two models can reveal the differences. Fig. 2 compares the remaining quantity of options in the basic model and the mechanism selection model in one of the selected scenarios. In this figure, the basic model selects the wholesale mechanism in period 3, while the mechanism selection model uses the option mechanism. Despite such slight differences, both models provide similar results. Table 7 presents the main outputs of the basic and the mechanism selection models. Following remarks could be extracted from Table 7 .

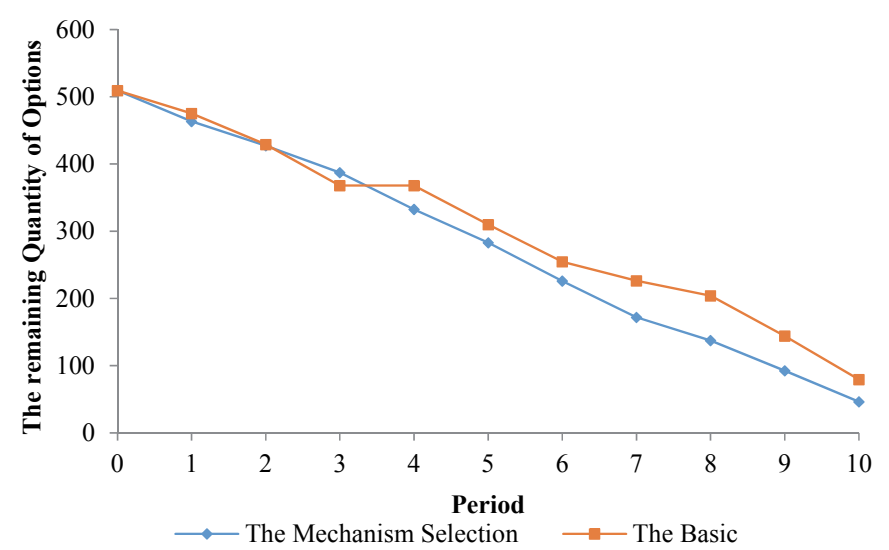

Fig. 2. Th remaining of the options in the basic model and the mechanism selection model

- Use of the mechanism selection model creates a higher profit for the members and the whole $\mathrm{SC}$. As the order quantities are equal, the reason for the higher profit is the different selected mechanisms in some periods, 
- The mechanism selection model remarkably reduces the average shortage. This model compares the shortage costs and the extra profit that the retailer will gain through using the wholesale mechanism. In other words, the retailer compares the shortage with a coefficient of $b$ and the extra profit gained by using the wholesale mechanism with coefficient of $W P_{s t}-$ $\left(e+o . e^{r t}\right)$. If the first one is bigger, the retailer chooses the option mechanism. Otherwise, the wholesale mechanism will be selected.

Table 7

Outputs of the basic model and the mechanism selection model

\begin{tabular}{lccccc}
\hline \multicolumn{1}{c}{ Model } & $\begin{array}{c}\text { Order } \\
\text { Quantity }\end{array}$ & $\begin{array}{c}\text { Retailer's } \\
\text { Profit }\end{array}$ & $\begin{array}{c}\text { Manufacturer's } \\
\text { Profit }\end{array}$ & $\begin{array}{c}\text { Total } \\
\text { Profit }\end{array}$ & $\begin{array}{c}\text { Average } \\
\text { shortage }\end{array}$ \\
\hline The basic & 509 & 1664.63 & 1285.748 & 2950.378 & 4.85 \\
The Mechanism Selection & 509 & 1691.507 & 1309.185 & 3000.692 & 1.6 \\
\hline
\end{tabular}

\section{Conclusions}

A coordination mechanism improves the profit and the stability of the SC. Although a perfect mechanism will be achieved if all the members of the chain act as a single organization, it is not always possible. Therefore, the academicians and the practitioners try to design coordination mechanisms in decentralized chains to avoid losses of decentralization as much as possible. In this study, a novel mixed coordination contract was introduced in which an American option mechanism and a wholesale mechanism were used in a retailer-manufacturer SC in a dynamic framework. The retailer as the leader adopted one of them according to a logical condition. The most crucial decision variable was the order quantity of the manufacturer, which was decided by a non-linear stochastic programming model in which the market demand and the market price were assumed to change over time, randomly. Moreover, a cooperative decentralized SC was addressed in which the retailer adopted the optimal order quantity of the centralized SC (chain-wide strategy). A scenario planning approach was used to treat random variables. Also, the number of generated scenarios was reduced with an optimal scenario reduction framework. In addition, a mechanism selection model was addressed in which the selection of the contract was considered a decision variable rather than a predetermined input. A numerical experiment demonstrated a remarkable improvement in the profit of both members in comparison with a wholesale contract. Also, the results showed that using a chain-wide strategy rather than the optimal solution of the proposed model can slightly increase the profit of the chain. To evaluate the effects of the option price and the exercise price, a sensitivity analysis was performed on them. As a result, the order quantity of the retailer was decreasing in both the option price and the exercise price. Similarly, this relation was confirmed between the profits of both members with the option and the exercise price. It was shown that there were some couples of option and exercise price, which provided a same profit for the SC. In essence, these couples formed a decreasing indifference curve along which the profit of the chain remains constant. In addition, because the mixed contract allowed the members to interact during a multi-period horizon, the interest rate played a substantial role in the problem. The higher the interest rate, the less total profit was gained by both members. Also, an increase in the interest rate reduced slightly the order quantity. This caused an additional decrease in the profit of the members and the whole chain. Finally, it was shown that the mechanism selection model had a better performance than the basic model. This modified version of the model avoided shortage more than the basic model, which increased the profit of the retailer. The proposed mixed contract is the first use of a kind of the American option mechanism in the coordination literature. It can be a new tool for multi-period contracts to use in the future studies. Also, using other types of the option mechanism can inspire the future studies to design a more effective coordination mechanism. Although the current study assumes that the manufacturer follows a MTO production policy, future studies can relax this assumption and uses a Stackelberg game to calculate the optimal production quantity of the manufacturer. 


\section{Appendix A:}

Proof of the optimal order and the optimal production strategy of the wholesale mechanism. In this mechanism, retailer's and manufacturer's strategies are independent. Therefore, optimal strategy for each of them should be provided and optimally conditions should be checked.

Firstly, expected values of Eq. (4) and Eq. (5) have been obtained in following equations:

$$
\begin{aligned}
& E\left(\Pi_{t s}^{R W}\right)=P_{t s}\left(\int_{-\infty}^{Q_{t s}^{W R}} x d F(x)+\int_{Q_{t s}^{W R}}^{+\infty} Q_{t s}^{W R} d F(x)\right)-W_{t s} Q_{t s}^{W R}-b \int_{Q_{t s}^{W R}}^{+\infty}(x- \\
& \left.Q_{t s}^{W R}\right) d F(x)+v \int_{-\infty}^{Q_{t s}^{W R}}\left(Q_{t s}^{W R}-x\right) d F(x) \\
& E\left(\Pi_{t s}^{R W}\right)=W_{t s}\left(\int_{-\infty}^{Q_{t s}^{W M}} x d F(x)+\int_{Q_{t s}^{W M}}^{+\infty} Q_{t s}^{W M} d F(x)\right)-c \cdot Q_{t s}^{W M}-b \int_{Q_{t s}^{W M}}^{+\infty}(x- \\
& \left.Q_{t s}^{W M}\right) d F(x)+v \int_{-\infty}^{Q_{t s}^{W M}}\left(Q_{t s}^{W M}-x\right) d F(x)
\end{aligned}
$$

Where $Q_{t s}^{W R}$ and $Q_{t s}^{W M}$ are retailer's order and manufacturer's production quantities, respectively in wholesale mechanism. To obtain optimal value of order quantity of the retailer, the first and the second order derivatives w. r. t. $Q_{t s}^{W R}$ have been provided in following equations:

$$
\begin{aligned}
& \frac{\partial E\left(\Pi_{t s}^{R W}\right)}{\partial Q_{t s}^{W R}}=P_{t s}+b-W_{t s}-\left(P_{t s}+b-v\right) F\left(Q_{t s}^{W R}\right)=0 \\
& \frac{\partial^{2} E\left(\Pi_{t s}^{R W}\right)}{\partial\left(Q_{t s}^{W R}\right)^{2}}=-\left(P_{t s}+b-v\right) f\left(Q_{t s}^{W R}\right)
\end{aligned}
$$

It is obvious that $P_{t s}>v$. Therefore, $-\left(P_{t s}+b-v\right)<0$. Accordingly, the second order derivative of the retailer's profit function is strictly concave. Therefore, the solution of the Eq. (A.3) is optimal value of the retailer's order quantity which has been obtained as $\widehat{Q}_{t s}^{W R}=F^{-1}\left(\frac{P_{t s}+b-W_{t s}}{P_{t s}+b-v}\right)$.

On the other hand, to obtain optimal production quantity of the manufacturer, the first and the second order derivatives w. r. t. $Q_{t s}^{W M}$ have been obtained in following equations:

$$
\begin{aligned}
& \frac{\partial E\left(\Pi_{t s}^{W M}\right)}{\partial Q_{t s}^{W M}}=W_{t s}+b-c-\left(W_{t s}+b-v\right) F\left(Q_{t s}^{W M}\right)=0 \\
& \frac{\partial^{2} E\left(\Pi_{t s}^{W M}\right)}{\partial\left(Q_{t s}^{W M}\right)^{2}}=-\left(W_{t s}+b-v\right) f\left(Q_{t s}^{W R}\right)
\end{aligned}
$$

Since $W_{t s}>v$, therefore, $-\left(W_{t s}+b-v\right)<0$. Accordingly, the second order derivative of the manufacturer's profit function is negative and Eq. (A.2) is strictly concave. So, solution of Eq. (A.5) as $\hat{Q}_{t s}^{W M}=F^{-1}\left(\frac{W_{t s}+b-c}{P_{t s}+b-v}\right)$ is the proven optimal production quantity of the manufacturer.

Proof of the optimal order quantity in the centralized SC. Firstly, expected value of profit function of the centralized $\mathrm{SC}$ has been presented in the following equation:

$$
E\left(\Pi_{S}^{S C}\right)=\bar{P} \cdot \int_{-\infty}^{Q^{S C}} y_{s} d F\left(y_{s}\right)+\int_{Q}^{+\infty} Q^{S C} d F\left(y_{s}\right)-c \cdot Q^{S C}-b \cdot \int_{Q}^{+\infty}\left(y_{s}-Q^{S C}\right) d F\left(y_{s}\right)
$$

Where, $y_{s}=\sum_{t=1}^{T} D_{t s}$. In terms of the hypothetical centralized supply chain, to obtain optimal quantity, the first and the second order derivatives of Eq. (A.7) have been presented in following equations:

$$
\begin{aligned}
& \frac{\partial E\left(\Pi_{S}^{S C}\right)}{\partial Q^{S C}}=\bar{P}+b-c-(\bar{P}+b) F\left(Q^{S C}\right)=0 \\
& \frac{\partial^{2} E\left(\Pi_{S}^{S C}\right)}{\partial\left(Q^{S C}\right)^{2}}=-(\bar{P}+b) f\left(Q^{S C}\right)
\end{aligned}
$$


520

Since $\bar{P}>0$ and $b>0$, so, $=-(\bar{P}+b) f\left(Q^{S C}\right)<0$. Accordingly, the second order derivative of the profit function of the hypothetical centralized supply chain is negative and Eq. (A.7) is strictly concave while it is sufficient optimally condition. Finally, the solution of Eq. (A.8) $\left(\hat{Q}^{S C}=F_{c}^{-1}\left(\frac{\bar{P}+b-c}{\bar{P}+b}\right)\right)$ is proven optimal quantity.

\section{Appendix B:}

The complete version of the scenario reduction model is presented here. See Li \& Floudas (2014) for more explanations.

$$
\min \sum_{s=1}^{S} \operatorname{Pr}_{s}^{\text {orig }} d_{s}+Z_{\text {max }}^{\text {err }}+Z_{\text {min }}^{\text {err }}+Z_{\text {exp }}^{\text {err }}
$$

subject to:

$$
\begin{aligned}
& \begin{array}{ll}
\sum_{s=1}^{S} y_{S}=N & \forall S
\end{array}
\end{aligned}
$$

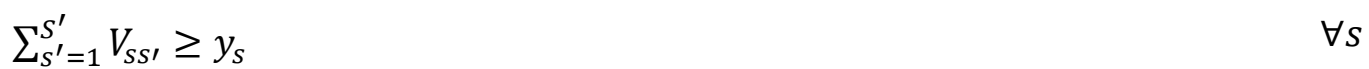

$$
\begin{aligned}
& 0 \leq V_{s S^{\prime}} \leq 1-y_{S^{\prime}} \quad \forall s, s^{\prime} \\
& d_{S}=\sum_{S^{\prime}=1}^{S^{\prime}} C_{S S^{\prime}} V_{S S^{\prime}} \quad \forall s \\
& 0 \leq d_{s} \leq y_{s} Z_{\text {max }}^{\text {orig }} \quad \forall s
\end{aligned}
$$

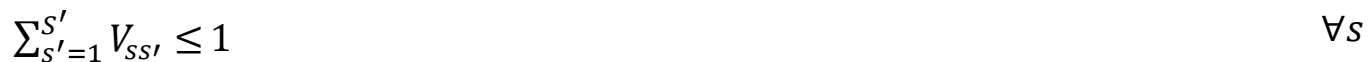

$$
\begin{aligned}
& \operatorname{Pr}_{S^{\prime}}^{\text {New }}=\left(1-y_{s^{\prime}}\right) \operatorname{Pr}_{s^{\prime}}^{\text {Orig }}+\sum_{s=1}^{S} \operatorname{Pr}_{S}^{\text {Orig }} V_{S S^{\prime}} \quad \forall s^{\prime} \\
& Z_{\text {exp }}^{N e w}=\sum_{s=1}^{S} P r_{S}^{N e w} Z_{s}^{*} \\
& Z_{\text {exp }}^{\text {err }} \geq Z_{\text {exp }}^{\text {New }}-Z_{\text {exp }}^{\text {Orig }} \\
& Z_{\text {exp }}^{\text {err }} \geq-Z_{\text {exp }}^{\text {New }}+Z_{\text {exp }}^{\text {orig }} \\
& y_{s}^{\max } \leq 1-y_{s} \quad \forall s \\
& \sum_{S=1}^{S} y_{S}^{\max }=1 \\
& Z_{\max }^{\text {New }}=\sum_{s=1}^{S} y_{s}^{\max } Z_{s}^{*} \\
& Z_{\text {max }}^{\text {New }} \geq\left(1-y_{s}\right) Z_{s}^{*}+y_{s} Z_{\text {min }}^{\text {Orig }} \\
& y_{s}^{\min } \leq 1-y_{s} \\
& \sum_{S=1}^{S} y_{s}^{\text {min }}=1 \\
& Z_{\text {min }}^{\text {New }}=\sum_{S=1}^{S} y_{s}^{\text {min }} Z_{S}^{*} \\
& Z_{\text {min }}^{\text {New }} \leq\left(1-y_{s}\right) Z_{s}^{*}+y_{s} Z_{\text {max }}^{\text {Orig }} \\
& Z_{\text {max }}^{\text {err }}=Z_{\text {max }}^{\text {Orig }}-Z_{\text {max }}^{\text {New }} \\
& Z_{\text {min }}^{\text {err }}=Z_{\text {min }}^{\text {Orig }}-Z_{\text {min }}^{\text {New }} \\
& y_{s}, y_{s}^{\max }, y_{s}^{\min } \in\{0,1\} \quad \forall s \\
& Z_{s}^{*}=\max \sum_{t=1}^{T}\left(P_{t s} \cdot \alpha_{t s}-e p \cdot \beta_{t s}-W_{t s} \cdot\left(1-Y_{t s}\right) \cdot D_{t s}-b \cdot \delta_{t s} \cdot e^{-r t}\right)-o \cdot Q \quad \forall s
\end{aligned}
$$




$$
\begin{aligned}
& Z_{\text {max }}^{\text {Orig }}=\max _{s} Z_{s}^{*} \\
& Z_{\text {min }}^{\text {Orig }}=\min _{s} Z_{s}^{*} \\
& Z_{\text {exp }}^{\text {Orig }}=\sum_{s=1}^{S} \operatorname{Pr}_{s} Z_{s}^{*}
\end{aligned}
$$

\section{References}

Arani, H. V., Rabbani, M., \& Rafiei, H. (2016). A revenue-sharing option contract toward coordination of supply chains. International Journal of Production Economics, 178, 42-56.

Babich, V. (2006). Vulnerable options in supply chains: Effects of supplier competition. Naval Research Logistics (NRL), 53(7), 656-673.

Bresnahan, T. F., \& Reiss, P. C. (1985). Dealer and manufacturer margins. The RAND Journal of Economics, 253-268.

Burnetas, A., \& Ritchken, P. (2005). Option pricing with downward-sloping demand curves: The case of supply chain options. Management Science, 51(4), 566-580.

Buzacott, J., Yan, H., \& Zhang, H. (2011). Risk analysis of commitment-option contracts with forecast updates. IIE Transactions, 43(6), 415-431.

Cai, J., Zhong, M., Shang, J., \& Huang, W. (2017). Coordinating VMI supply chain under yield uncertainty: Option contract, subsidy contract, and replenishment tactic. International Journal of Production Economics, 185, 196-210.

Chen, X., Hao, G., \& Li, L. (2014). Channel coordination with a loss-averse retailer and option contracts. International Journal of Production Economics, 150, 52-57.

Chen, X., \& Shen, Z. J. (2012). An analysis of a supply chain with options contracts and service requirements. IIE Transactions, 44(10), 805-819.

Chen, X., Wan, N., \& Wang, X. (2017). Flexibility and coordination in a supply chain with bidirectional option contracts and service requirement. International Journal of Production Economics, 193, 183192.

Cheng, F., Ettl, M., Lin, G. Y., Schwarz, M., \& Yao, D. D. (2003). Flexible supply contracts via options. IBM TJ Watson Research Center Working Paper.

Chopra, S., \& Meindl, P. (2007). Supply chain management. Strategy, planning \& operation. Das summa summarum des management, 265-275.

Dupačová, J., Gröwe-Kuska, N., \& Römisch, W. (2003). Scenario reduction in stochastic programming. Mathematical programming, 95(3), 493-511.

Gan, X., Sethi, S. P., \& Yan, H. (2005). Channel coordination with a risk-neutral supplier and a downside-risk-averse retailer. Production and Operations Management, 14(1), 80-89.

Gomez_Padilla, A., \& Mishina, T. (2009). Supply contract with options. International Journal of Production Economics, 122(1), 312-318.

Gröwe-Kuska, N., Heitsch, H., \& Romisch, W. (2003, June). Scenario reduction and scenario tree construction for power management problems. In Power tech conference proceedings, 2003 IEEE Bologna (Vol. 3, pp. 7-pp). IEEE.

Hasani, R., Jafarzadeh, H., \& Khoshalhan, F. (2013). A new method for supply chain coordination with credit option contract and customers' backordered demand. Uncertain Supply Chain Management, 1(4), 207-218.

Heitsch, H., \& Römisch, W. (2003). Scenario reduction algorithms in stochastic programming. Computational optimization and applications, 24(2-3), 187-206.

Huang, M. G. (2009). Real options approach-based demand forecasting method for a range of products with highly volatile and correlated demand. European journal of operational research, 198(3), 867877.

Hull, J. C., \& Basu, S. (2016). Options, futures, and other derivatives. Pearson Education India. 
Jörnsten, K., Nonås, S. L., Sandal, L., \& Ubøe, J. (2013). Mixed contracts for the newsvendor problem with real options and discrete demand. Omega, 41(5), 809-819.

Kanda, A., \& Deshmukh, S. G. (2008). Supply chain coordination: perspectives, empirical studies and research directions. International journal of production Economics, 115(2), 316-335.

Lee, H. L., Padmanabhan, V., \& Whang, S. (1997). Information distortion in a supply chain: The bullwhip effect. Management science, 43(4), 546-558.

Li, H., Ritchken, P., \& Wang, Y. (2009). Option and forward contracting with asymmetric information: Valuation issues in supply chains. European Journal of Operational Research, 197(1), 134-148.

Li, Z., \& Floudas, C. A. (2014). Optimal scenario reduction framework based on distance of uncertainty distribution and output performance: I. Single reduction via mixed integer linear optimization. Computers \& Chemical Engineering, 70, 50-66.

Liu, C., Jiang, Z., Liu, L., \& Geng, N. (2013). Solutions for flexible container leasing contracts with options under capacity and order constraints. International Journal of Production Economics, 141(1), 403-413.

Luo, M., Li, G., Wan, C. J., Qu, R., \& Ji, P. (2015). Supply chain coordination with dual procurement sources via real-option contract. Computers \& Industrial Engineering, 80, 274-283.

Niknam, T., Azizipanah-Abarghooee, R., \& Narimani, M. R. (2012). An efficient scenario-based stochastic programming framework for multi-objective optimal micro-grid operation. Applied Energy, 99, 455-470.

Nomikos, N. K., Kyriakou, I., Papapostolou, N. C., \& Pouliasis, P. K. (2013). Freight options: Price modelling and empirical analysis. Transportation Research Part E: Logistics and Transportation Review, 51, 82-94.

Tsay, A. A. (1999). The quantity flexibility contract and supplier-customer incentives. Management science, 45(10), 1339-1358.

Wang, X., \& Liu, L. (2007). Coordination in a retailer-led supply chain through option contract. International Journal of Production Economics, 110(1), 115-127.

Wang, Y. (2010). Scenario reduction heuristics for a rolling stochastic programming simulation of bulk energy flows with uncertain fuel costs. Iowa State University.

Watkins, D. W., McKinney, D. C., Lasdon, L. S., Nielsen, S. S., \& Martin, Q. W. (2000). A scenariobased stochastic programming model for water supplies from the highland lakes. International Transactions in Operational Research, 7(3), 211-230.

Xia, Y., Ramachandran, K., \& Gurnani, H. (2011). Sharing demand and supply risk in a supply chain. IIE Transactions, 43(6), 451-469.

$\mathrm{Xu}, \mathrm{H}$. (2010). Managing production and procurement through option contracts in supply chains with random yield. International Journal of Production Economics, 126(2), 306-313.

Yang, L., Tang, R., \& Chen, K. (2017). Call, put and bidirectional option contracts in agricultural supply chains with sales effort. Applied Mathematical Modelling, 47, 1-16.

Zhao, Y., Ma, L., Xie, G., \& Cheng, T. E. (2013). Coordination of supply chains with bidirectional option contracts. European Journal of Operational Research, 229(2), 375-381.

Zhao, Y., Wang, S., Cheng, T. E., Yang, X., \& Huang, Z. (2010). Coordination of supply chains by option contracts: A cooperative game theory approach. European Journal of Operational Research, 207(2), 668-675.

Zhang, R. Q., \& Wang, R. P. (2009). Scenario-based stochastic capacity planning model and decision risk analysis. Systems Engineering-Theory \& Practice, 29(1), 55-63.

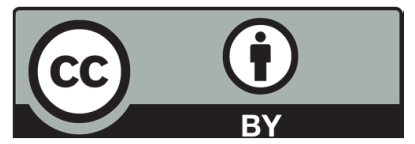

(C) 2018 by the authors; licensee Growing Science, Canada. This is an open access article distributed under the terms and conditions of the Creative Commons Attribution (CC-BY) license (http://creativecommons.org/licenses/by/4.0/). 